\title{
ON PAIRWISE GENERALIZED ALPHA SEPARATION AXIOMS IN INTUITIONISTIC BIFUZZY TOPOLOGICAL SPACES
}

\author{
C.S. Gowri ${ }^{1}$ § D. Kalamani ${ }^{2}$, R. Dhavaseelan ${ }^{3}$ \\ ${ }^{1}$ Department of Mathematics \\ Velalar College of Engineering and Technology \\ Erode, Tamil Nadu, INDIA \\ ${ }^{2}$ Department of Mathematics \\ Kongu Engineering College \\ Perundurai, Tamil Nadu, INDIA \\ ${ }^{3}$ Department of Mathematics \\ Sona College of Technology \\ Salem, Tamil Nadu, INDIA
}

\begin{abstract}
The aim of this work is to investigate several types of pairwise $G \alpha$ $T_{K}, K=0,1,2, \ldots$ separation axioms in Intuitionistic bifuzzy topological spaces, based on the characterizations of pairwise separation axioms in bitopological spaces. Intuitionistic bifuzzy pairwise $G \alpha$-regular spaces, pairwise $G \alpha$-normal spaces are also studied and their relationships with other separation axioms are discussed.
\end{abstract}

AMS Subject Classification: 54D10, 54E55, 03E72

Key Words: intuitionistic bifuzzy topological spaces, intuitionistic bifuzzy pairwise $G \alpha T_{K}, K=0,1,2, \ldots$ separation axioms, intuitionistic bifuzzy pairwise $G \alpha$-regular spaces, intuitionistic bifuzzy pairwise $G \alpha$-normal spaces

\section{Introduction}

The triple $\left(X, \tau_{1}, \tau_{2}\right)$, where $X$ is a non empty set and $\tau_{1}, \tau_{2}$ are topologies on $X$ is called a bitopological space. Two topologies on the same set may separate the

Received: August 30, 2013

(C) 2014 Academic Publications, Ltd.

$\S$ Correspondence author url: www.acadpubl.eu 
points of the set to a different degree. J.C. Kelly [13] initiated the systematic study of such spaces and several other authors have contributed to the subsequent development of various bitopological properties. Zadeh [15] introduced the concept of fuzzy sets. After that there have been a number of generalizations of this fundamental concept. Atanassov [1] introduced the notion of intuitionistic fuzzy sets. Using the notion of intuitionistic fuzzy sets, Coker [7] introduced the notion of intuitionistic fuzzy topological spaces. The separation axioms stipulate the degree in which distinct points or closed sets are separated by open sets. These axioms are statements about the richness of the topology. Fuzzy separation axioms and fuzzy continuity in fuzzy bitopological spaces was given by A.S. Abu Safiya, A. A. Fora, and M. W. Warner [3]. Pairwise separation axioms in IFTS were introduced by Sadik Bayhan and Dogan Coker $[5,6]$. It is the aim of this paper to give more properties of pairwise separation axioms introduced by Sadik Bayhan. In chapter [3] Intuitionistic bifuzzy pairwise $G \alpha T_{K}$ spaces is introduced and their interrelationship are studied. In chapter [4] Intuitionistic bifuzzy pairwise $G \alpha$-regular spaces, Intuitionistic bifuzzy pair wise $G \alpha$-normal spaces are studied as a development of pairwise separation axioms in bitopological spaces by T.M. Nour. We shall use $P$ - to denote pairwise, $P-G \alpha T_{K}$ spaces for pairwise generalized alpha $T_{K}$ spaces, $K=0,1,2, \ldots$ $P$-regular to denote pairwise regular and $P$-normal to denote pairwise normal.

\section{Preliminaries}

Definition 2.1. [1] Let $X$ be a non empty fixed set. An intuitionistic fuzzy set (IFS in short) $A$ in $X$ is an object having the form $A=\left\{\left\langle x, \mu_{A}(x), \gamma_{A}(x)\right\rangle\right.$ : $x \in X\}$ where the functions $\mu_{A}: X \longrightarrow[0,1]$ and $\gamma_{A}: X \longrightarrow[0,1]$ denote the degree of membership (namely $\mu_{A}(x)$ ) and the degree of nonmembership (namely $\gamma_{A}(x)$ ) for each element $x \in X$ to the set $A$ respectively, and $0 \leq$ $\mu_{A}(x)+\gamma_{A}(x) \leq 1$ for each $x \in X$.

Denote by $\operatorname{IFS}(X)$, the set of all intuitionistic fuzzy sets in $X$.

Definition 2.2. [7] An intuitionistic fuzzy topology (IFT in short)on $X$ is a family $\tau$ of intuitionistic fuzzy sets in $X$ satisfying the following axioms

(i) $0 \sim, 1 \sim \in \tau$;

(ii) $G_{1} \cap G_{2} \in \tau$ for any $G_{1}, G_{2} \in \tau$;

(iii) $\cup G_{i} \in \tau$ for arbitrary family $\left\{G_{i} \mid i \in I\right\} \subseteq \tau$. 
In this case the pair $(X, \tau)$ is called an intuitionistic fuzzy topological space (IFTS in short) and any intuitionistic fuzzy sets in $\tau$ is known as an intuitionistic fuzzy open $\operatorname{set}(I F O S$ in short) in $X$.

Definition 2.3. [1] Let $A$ and $B$ be IFS's of the form $A=\left\{\left\langle x, \mu_{A}(x), \gamma_{A}(x)\right\rangle\right.$ : $x \in X\}$ and $B=\left\{\left\langle x, \mu_{B}(x), \gamma_{B}(x)\right\rangle: x \in X\right\}$. Then

(a) $A \subseteq B$ iff $\mu_{A}(x) \leq \mu_{B}(x)$ and $\gamma_{A}(x) \geq \gamma_{B}(x)$ for all $x \in X$

(b) $A=B$ if and only if $A \subseteq B$ and $B \subseteq A$

(c) $\bar{A}=\left\{\left\langle x, \gamma_{A}(x), \mu_{A}(x)\right\rangle: x \in X\right\}$

(d) $A \cap B=\left\{\left\langle x, \mu_{A}(x) \wedge \mu_{B}(x), \gamma_{A}(x) \vee \gamma_{B}(x)\right\rangle: x \in X\right\}$

(e) $A \cup B=\left\{\left\langle x, \mu_{A}(x) \vee \mu_{B}(x), \gamma_{A}(x) \wedge \gamma_{B}(x)\right\rangle: x \in X\right\}$

(f) $0_{\sim}=\{\langle x, 0,1\rangle: x \in X\}$ and $1 \sim=\{\langle x, 1,0\rangle: x \in X\}$

Definition 2.4. [12] An IFS A of an $\operatorname{IFTS}(X, \tau)$ is said to be an intuitionistic fuzzy generalized $\alpha$ closed set $(I F G \alpha C S$ in short) if $\alpha c l(A) \subseteq U$ whenever $A \subseteq U$ and $U$ is an $I F \alpha O S$ in $X$.

Definition 2.5. [4] Let $X$ be a non-empty set and $x \in X$, a fixed element in $X$. If $\alpha \in(0,1)$ and $\beta \in(0,1)$ are fixed real number such that $\alpha+\beta \leq 1$ then the $\operatorname{IFS} x_{(\alpha, \beta)}=\left(x_{\alpha}, 1-x_{(1-\beta)}\right)$ is called an intuitionistic fuzzy point in $X$ (IFP, in short) in $X$, and $x$ is called its support. Two IFPs are said to be distinct if there supports are distinct.

Let $x_{(\alpha, \beta)}$ be an IFP in $X$ and $A=\left(\mu_{A}, \gamma_{A}\right)$ be an IFS in $X$ then $x_{(\alpha, \beta)}$ is belong to $A$ if $\alpha \leq \mu_{A}(x), \gamma_{A} \geq \gamma_{A}(x)$.

Definition 2.6. [5] Let $\langle a, b\rangle,\left\langle a_{1}, b_{1}\right\rangle,\left\langle a_{2}, b_{2}\right\rangle$ and $\left\langle a_{i}, b_{i}\right\rangle(i \in J)$ be intuitionistic fuzzy pairs. Then we define

(i) $\left\langle a_{1}, b_{1}\right\rangle \leq\left\langle a_{2}, b_{2}\right\rangle$ if and only if $a_{1} \leq a_{2}$ and $b_{1} \geq b_{2}$,

(ii) $\left\langle a_{1}, b_{1}\right\rangle=\left\langle a_{2}, b_{2}\right\rangle$ if and only if $a_{1}=a_{2}$ and $b_{1}=b_{2}$,

(iii) if $\vee\left\langle a_{i}, b_{i}\right\rangle=\left\langle\vee a_{i}, \wedge b_{i}\right\rangle$ and $\wedge\left\langle a_{i}, b_{i}\right\rangle=\left\langle\wedge a_{i}, \vee b_{i}\right\rangle$,

(iv) $\overline{\langle a, b\rangle}=\langle b, a\rangle$,

(v) $1^{\sim}=\langle 1,0\rangle$ and $0^{\sim}=\langle 0,1\rangle$.

Definition 2.7. [4] Let $\left(X, \tau_{1}, \tau_{2}\right)$ be a bitopological space. Then it is called 
(i) pairwise $T_{0}$ if for all $x$ and $y$ in $X$, there exist $U$ in $\tau_{1}$ with $x \in U, y \notin U$ or there exist $V$ in $\tau_{2}$ with $y \in V, x \notin V$.

(ii) pairwise $T_{1}$ if for all $x$ and $y$ in $X$, there exist $U$ in $\tau_{1}$ with $x \in U, y \notin U$ and there exist $V$ in $\tau_{2}$ with $y \in V, x \notin V$.

(iii) pairwise $T_{2}$ if and only if for each pair of distinct fuzzy points $x_{r, s}$ and $y_{p, q}$ in $X$, there exist $U$ in $\tau_{1}$ with $x_{r, s} \in U$ and there exist $V$ in $\tau_{2}$ with $y_{p, q} \in V$ such that $U \cap V=0_{\sim}$.

Definition 2.8. [4] An IFTS $(X, \tau)$ is called

(i) $T_{0}$ if for all $x, y \in X, X \neq y, \exists U=\left(\mu_{U}, \gamma_{U}\right), V=\left(\mu_{V}, \gamma_{V}\right) \in \tau$ such that $U(x)=\tilde{1}, U(y)=\tilde{0}$ or $V(y)=\tilde{1}, V(x)=\tilde{0}$.

(ii) $T_{1}$ for all $x, y \in X, X \neq y, \exists U=\left(\mu_{U}, \gamma_{U}\right), V=\left(\mu_{V}, \gamma_{V}\right) \in \tau$ such that $U(x)=\tilde{1}, U(y)=\tilde{0}$ and $V(y)=\tilde{1}, V(x)=\tilde{0}$.

(iii) $T_{2}$ if for all pair of distinct intuitionistic fuzzy points $x_{r, s}$ and $y_{p, q}$ in $X$, $\exists U=\left(\mu_{U}, \gamma_{U}\right), V=\left(\mu_{V}, \gamma_{V}\right) \in \tau$ such that $x_{r, s} \in U, y_{p, q} \in V$ and $U \cap V=$ 0 .

Definition 2.9. [14] If $\left\{A_{i}=\left(\mu_{A_{\mathrm{i}}}, \gamma_{A_{\mathrm{i}}}\right), i \in J\right\}$ is a family of $I F S \mathrm{~s}$ in $X_{i}$, then their product is defined as the IFS in $\prod X_{i}$ given by $\prod A_{i}=$ $\left(\prod \mu_{A_{\mathrm{i}}}, \prod^{*} \gamma_{A_{\mathrm{i}}}\right)$ where $\prod \mu_{A_{\mathrm{i}}}=\inf \left(\mu_{A_{\mathrm{i}}}\left(x_{i}\right)\right) \forall x=\prod x_{i} \in X$ and $\prod^{*} \gamma_{A_{\mathrm{i}}}=$ $\sup \left(\gamma_{A_{\mathrm{i}}}\left(x_{i}\right)\right)$.

Definition 2.10. [4] An $\operatorname{IFS} N$ in an $\operatorname{IFT} S(X, \tau)$ is called an intuitionistic fuzzy neighbourhood ( $I F N$, in short) of an $I F P x_{(\alpha, \beta)}$ if $\exists U \in \tau$ such that $x_{(\alpha, \beta)} \in U \subseteq N$.

Proposition 2.1. [4] Let $(X, \tau)$ be an IFTS. Then an IFS $A$ in $X$ is an $I F O S$ if and only if $A$ is an $I F N$ of each of $I F P x_{(\alpha, \beta)} \in A$.

Proposition 2.2. [4] Let $\left(X, \tau_{1}, \tau_{2}\right)$ be a BFTS space $\tau_{\left(\tau_{1}, \tau_{2}\right)}=\left\{\left(U, V^{c}\right) \mid U \in \tau_{1}, V \in \tau_{2}\right.$ and $\left.\left.U \subseteq V\right)\right\}$. Then $(X, \tau)$ is an IFTS and $\left(\tau_{\left(\tau_{1}, \tau_{2}\right)}\right)_{1}=\tau_{1},\left(\tau_{\left(\tau_{1}, \tau_{2}\right)}\right)_{1}=\tau_{2}$.

Remark 2.1. [4] Let $(X, \tau)$ be an IFTS and let $\tau_{1}=\left\{\mu_{A} \mid \exists \gamma_{A} \in\right.$ $I^{X}$ such that $\left.\left(\mu_{A}, \gamma_{A}\right) \in \tau\right\}, \tau_{2}=\left\{1_{\sim}-\gamma_{A} \mid \exists \mu_{A} \in I^{X}\right.$ such that $\left.\left(\mu_{A}, \gamma_{A}\right) \in \tau\right\}$. 


\section{Pairwise Generalized Alpha Separation Axioms in Intuitionistic BiFuzzy Topological Spaces}

Definition 3.1. Let $X$ be a nonempty set and $\tau_{1}, \tau_{2}$ be two intuitionistic fuzzy topologies on $X$. Then $\left(X, \tau_{1}, \tau_{2}\right)$ is called an intuitionistic bifuzzy topological space(IBFTS, in short).

Definition 3.2. An $\operatorname{IFTS}(X, \tau)$ is called

(i) $G \alpha T_{0}$ if for all $x, y \in X, x \neq y$, there exist $G \alpha$-open sets $U=\left(\mu_{U}, \gamma_{U}\right), V=$ $\left(\mu_{V}, \gamma_{V}\right) \in \tau$ such that $U(x)=\tilde{1}, U(y)=\tilde{0}$ or $V(y)=\tilde{1}, V(x)=\tilde{0}$.

(ii) $G \alpha T_{1}$ for all $x, y \in X, X \neq y$, there exist $G \alpha$-open sets $U=\left(\mu_{U}, \gamma_{U}\right), V=$ $\left(\mu_{V}, \gamma_{V}\right) \in \tau$ such that $U(x)=\tilde{1}, U(y)=\tilde{0}$ and $V(y)=\tilde{1}, V(x)=\tilde{0}$.

(iii) $G \alpha T_{2}$ if for all pair of distinct intuitionistic fuzzy points $x_{r, s}$ and $y_{p, q}$ in $X$, there exist $G \alpha$-open sets $U=\left(\mu_{U}, \gamma_{U}\right), V=\left(\mu_{V}, \gamma_{V}\right) \in \tau$ such that $x_{r, s} \in U, y_{p, q} \in V$ and $U \cap V=0 \sim$.

Definition 3.3. (Pairwise $G \alpha T_{K}$ Spaces) An $\operatorname{IBFTS}\left(X, \tau_{1}, \tau_{2}\right)$ is called Pairwise $G \alpha T_{K}, K=0,1,2$ if for any pair of distinct points $x$ and $y$ in

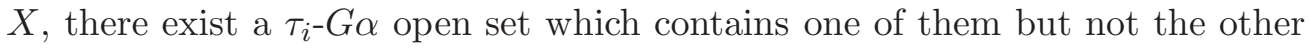
$i=1,2$.

Definition 3.4. An $\operatorname{IBFTS}\left(X, \tau_{1}, \tau_{2}\right)$ is called

(i) pairwise $G \alpha T_{0}$ if for all $x, y \in X, X \neq y$, there exist $\tau_{i}$-IFG $\alpha$-open set $U=\left(\mu_{U}, \gamma_{U}\right)$ and $\tau_{j}$-IFG $\alpha$-open set $V=\left(\mu_{V}, \gamma_{V}\right)$ such that $U(x)=$ $\tilde{1}, U(y)=\tilde{0}$ or $V(y)=\tilde{1}, V(x)=\tilde{0}, i, j=1,2, i \neq j$.

(ii) pairwise $G \alpha T_{1}$ for all $x, y \in X, x \neq y$, there exist $\tau_{i}$-IFG $\alpha$-open set $U=\left(\mu_{U}, \gamma_{U}\right)$ and $\tau_{j}$-IFG $\alpha$-open set $V=\left(\mu_{V}, \gamma_{V}\right)$ such that $U(x)=$ $\tilde{1}, U(y)=\tilde{0}$ and $V(y)=\tilde{1}, V(x)=\tilde{0}, i, j=1,2, i \neq j$.

(iii) pairwise $G \alpha T_{2}$ if for any pair of distinct points $x_{r, s}$ and $y_{p, q}$ in $X$, there exist $\tau_{i}-I F G \alpha$-open set $U$ and $\tau_{j}-I F G \alpha$-open set $V$ such that $x_{r, s} \in U, y_{p, q} \in$ $V$ and

$U \cap V=0_{\sim}, i, j=1,2, i \neq j$.

Proposition 3.1. Let $\left\{\left(X_{i}, \tau_{1 i}, \tau_{2 i}\right): i \in J\right\}$ be a family of IBFTS and $\left(\prod X_{i}, \prod \tau_{1 i}, \prod \tau_{2 i}\right)$ be their product IBFTS. Then $\left\{\left(\prod X_{i}, \prod \tau_{1 i}, \prod \tau_{2 i}\right): i \in\right.$

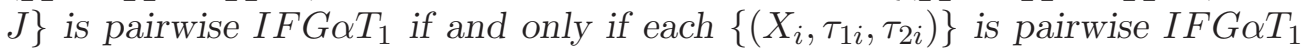
for all $i \in J$. 
Proof. Assume that $\left\{\left(X_{i}, \tau_{1 i}, \tau_{2 i}\right): i \in J\right\}$ be pairwise $I F G \alpha T_{1}$. To show that

$\left\{\left(\prod X_{i}, \prod \tau_{1 i}, \prod \tau_{2 i}\right): i \in J\right\}$ is pairwise $I F G \alpha T_{1}$. Choose $x, y \in X$ such that $x \neq y$. Let $x=\prod x_{j}, y=\prod y_{j}$ then $x_{j} \neq y_{j}$ for some $j \in J$. Now consider $x_{j}, y_{j} \in X_{j}$. Since $\left\{\left(X_{i}, \tau_{1 i}, \tau_{2 i}\right): i \in J\right\}$ is pairwise $I F G \alpha T_{1}$ there exists intuitionistic fuzzy generalized alpha open sets $U_{j}=\left(\mu_{U_{\mathrm{j}}}, \gamma_{U_{\mathrm{j}}}\right) \in \tau_{1 j}$ and $V_{j}=$ $\left(\mu_{V_{\mathrm{j}}}, \gamma_{V_{\mathrm{j}}}\right) \in \tau_{2 j}$ such that $U_{j}\left(x_{j}\right)=\tilde{1}, U_{j}\left(y_{j}\right)=\tilde{0}$ and $V_{j}\left(y_{j}\right)=\tilde{1}, V_{j}\left(x_{j}\right)=\tilde{0}$. Now consider the basic intuitionistic fuzzy generalized alpha open sets $U=\overline{\prod U_{i}}$ and $V=\overline{\prod V_{i}}$ where $U_{i}=V_{i}=(\tilde{1}, \tilde{0})$ for $i \neq j$ and $\overline{U_{j}}=U$ and $\overline{V_{j}}=V$ for $i=j$. Then $U=\prod \tau_{1 i}$ and $V=\prod \tau_{2 i}$ and hence $U(x)=\tilde{1}, U(y)=\tilde{0}$ and $V(y)=\tilde{1}, V(x)=\tilde{0}$. Hence $\left\{\left(\prod X_{i}, \prod \tau_{1 i}, \prod \tau_{2 i}\right): i \in J\right\}$ is pairwise $I F G \alpha T_{1}$.

Conversely let $\left\{\left(\prod X_{i}, \prod \tau_{1 i}, \prod \tau_{2 i}\right): i \in J\right\}$ is pairwise $I F G \alpha T_{1}$. To prove that $\left\{\left(X_{i}, \tau_{1 i}, \tau_{2 i}\right): i \in J\right\}$ is pairwise $I F G \alpha T_{1}$. Let $x_{i}, y_{i}$ then $x_{i} \neq y_{i}$. Now consider $x, y \in X$ such that $x=\prod x_{j}, y=\prod y_{j}$ where $x_{j}=y_{j}, j \neq i$ and $x_{i}=$ $x_{j}, y_{i}=y_{j}$ for $i \neq j$. Then $x \neq y$, since $\left\{\left(\prod X_{i}, \prod \tau_{1 i}, \prod \tau_{2 i}\right): i \in J\right\}$ is pairwise $I F G \alpha T_{1}$, there exists $U=\left(\mu_{U}, \gamma_{U}\right) \in \tau_{1}$ and $V=\left(\mu_{V}, \gamma_{V}\right) \in \tau_{2}$ such that $U(x)=\tilde{1}, U(y)=\tilde{0}$ and $V(y)=\tilde{1}, V(x)=\tilde{0}$. Consider the $I F P x_{r, s} \in U$ and $y_{p, q} \in V$. Then there exist basic intuitionistic fuzzy generalized alpha open sets $\prod U_{j} \in \tau_{1 j}$ and $\prod V_{j} \in \tau_{j}$ in $X$ such that $x_{r, s} \in \prod U_{j} \subseteq U$ and $y_{p, q} \in \prod V_{j} \subseteq V$. Since $x_{r, s} \in \prod U_{j} \subseteq U, U(x)=\tilde{1} \Rightarrow \prod U_{j}(x)=\tilde{1} \Rightarrow\left(\inf \mu_{U_{\mathrm{j}}}, \sup \gamma_{U_{\mathrm{j}}}\right)\left(x_{j}\right)=$ $\tilde{1} \Rightarrow r \leq \inf \mu_{U_{\mathrm{j}}}\left(x_{j}\right), s \geq \sup \gamma_{U_{\mathrm{j}}}\left(x_{j}\right)$ implies $r \leq \mu_{U_{\mathrm{j}}}\left(x_{j}\right), s \geq \gamma_{U_{\mathrm{j}}}\left(x_{j}\right)$ and $U(y)=\tilde{0}$ implies $\prod U_{j}(y)=\tilde{0}$.

Similarly $y_{p, q} \in \prod V_{j} \subseteq V \Rightarrow p \leq \inf \mu_{V_{\mathrm{j}}}\left(y_{j}\right), q \geq \sup \gamma_{V_{\mathrm{j}}}\left(y_{j}\right)$. Also, since, $x_{j}=y_{j}$ for $i \neq j$. From the above we have $r \leq \mu_{U_{\mathrm{i}}}\left(x_{i}\right), s \geq \gamma_{U_{\mathrm{i}}}\left(x_{i}\right) \forall i \in J, i \neq$ $j, p \leq \mu_{V_{\mathrm{i}}}\left(y_{i}\right), q \geq \gamma_{V_{\mathrm{i}}}\left(y_{i}\right) \forall i \in J, i \neq j$. Therefore, $U(y)=\tilde{0}$ implies $\prod U_{j}(y)=$ $\tilde{0} \Rightarrow \inf \mu_{U_{\mathrm{j}}}\left(y_{j}\right)=0, \sup \gamma_{U_{\mathrm{j}}}\left(y_{j}\right)=1$ implies $\mu_{U_{\mathrm{j}}}\left(y_{j}\right)=0, \gamma_{U_{\mathrm{j}}}\left(y_{j}\right)=1$. This implies $\sup \mu_{U_{\mathrm{j}}}\left(y_{j}\right)=0$, inf $\gamma_{U_{\mathrm{j}}}\left(y_{j}\right)=1$. That is, $U_{j}\left(y_{j}\right)=\tilde{0}$. Similarly we can show that $V_{j}\left(x_{j}\right)=\tilde{0}$. Thus we have $U_{i}\left(x_{i}\right) \geq r, U_{i}\left(y_{i}\right)=\tilde{0}$ and $V_{i}\left(y_{i}\right) \geq$ $p, V_{i}\left(x_{i}\right)=\tilde{0}$. Consider, $\sup U_{i}=U_{i} \in \tau_{1 i}$ and $\sup V_{i}=V_{i} \in \tau_{2 i}$. Then $U_{i}\left(x_{i}\right)=\tilde{1}, U_{i}\left(y_{i}\right)=\tilde{0}, V_{i}\left(x_{i}\right) \tilde{0}, V_{i}\left(y_{i}\right)=\tilde{1}$ showing that $\left(X_{i}, \tau_{1 i}, \tau_{2 i}\right)$ is pairwise IFG $\alpha T_{1}$

Theorem 3.1. Let $\left\{\left(X_{i}, \tau_{1 i}, \tau_{2 i}\right): i \in J\right\}$ be a family of IBFTS and $\left(\prod X_{i}, \prod \tau_{1 i}, \prod \tau_{2 i}\right)$ be their product IBFTS. Then $\left\{\left(\prod X_{i}, \prod \tau_{1 i}, \prod \tau_{2 i}\right): i \in\right.$ $J\}$ is pairwise IFGoT if and only if each $\left\{\left(X_{i}, \tau_{1 i}, \tau_{2 i}\right)\right\}$ is pairwise $I F G \alpha T_{0}$ for all $i \in J$.

Proposition 3.2. If an $\operatorname{IFTS}(X, \tau)$ is $\operatorname{IFG} \alpha T_{1}$ then $\left(X, \tau, \tau_{2}\right)$ is pairwise IFG $\alpha T_{1}$. 
Proof. Let $(X, \tau)$ be an $I F G \alpha T_{1}$ space. Then for all $x, y \in X, x \neq y$ there exist $\tau_{1}-I F G \alpha$-open set $U=\left(\mu_{U}, \gamma_{U}\right)$ and $\tau_{2}-I F G \alpha$-open set $V=\left(\mu_{V}, \gamma_{V}\right)$ such that $U(x)=\tilde{1}, U(y)=\tilde{0}$ and $V(y)=\tilde{1}, V(x)=\tilde{0}$. Since $U(x)=\tilde{1} \Rightarrow$ $\mu_{U}(x)=1, \gamma_{U}(x)=0, U(y)=\tilde{0} \Rightarrow \mu_{U}(y)=0, \gamma_{U}(y)=1$. Similarly, $V(y)=$ $\tilde{1} \Rightarrow \mu_{V}(y)=1, \gamma_{V}(y)=0, V(x)=\tilde{0} \Rightarrow \mu_{V}(y)=0, \gamma_{V}(x)=1$. Now $\mu_{U}(x)=$ $1 \Rightarrow x \in \mu_{U} \in \tau_{1}$ and $1 \sim-\gamma_{V}(x)=0 \Rightarrow x \in\left(1 \sim-\gamma_{V}\right) \in \tau_{2}$ and also $\mu_{U}(x)=1, \mu_{U}(y)=0,1_{\sim}-\gamma_{V}(x)=0$ and $1_{\sim}-\gamma_{V}(x)=0$ showing that $\left(X, \tau, \tau_{2}\right)$ is pairwise $I F G \alpha T_{1}$.

Proposition 3.3. Every IBF Pairwise $G \alpha T_{2}$ is IBF Pairwise $G \alpha T_{1}$.

Proof. Suppose that $\left(X, \tau, \tau_{2}\right)$ is Pairwise $G \alpha T_{2}$. Given any points $x_{r, s}$ and $y_{p, q}$ in $X$, there exist a $\tau_{1}-I F G \alpha$-open set $U$ containing $x_{r, s}$ and there exist a $\tau_{2}$-IFG $\alpha$-open set $V$ containing $y_{p, q}$ such that $U \cap V=0_{\sim}$. This implies $y_{p, q} \notin U$ and $x_{r, s} \notin V$. Hence $\left(X, \tau, \tau_{2}\right)$ is Pairwise Pairwise $G \alpha T_{1}$.

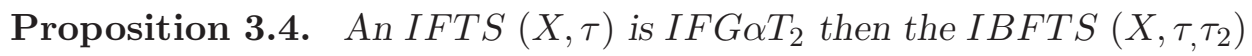
is pairwise $I F G \alpha T_{2}$.

Proof. Given $(X, \tau)$ be an $I F G \alpha T_{1}$ space. To prove that $\left(X, \tau, \tau_{2}\right)$ is pairwise $I F G \alpha T_{2}$. Let $x_{r}, y_{1-p}$ be any two $F P$ in $X$. Choose $(s, q) \in(0,1)$, then $x_{r, s}$ and $y_{p, q}$ are district $I F P$ 's in $X$. Since $(X, \tau)$ is $I F G \alpha T_{2}$ space there exist $G \alpha$-open sets $U=\left(\mu_{U}, \gamma_{U}\right), V=\left(\mu_{V}, \gamma_{V}\right) \in \tau$ such that $x_{r, s} \in U, y_{p, q} \in$ $V$ and $U \cap V=0_{\sim}$. Let $U=\left(\mu_{U}, \gamma_{U}\right), V=\left(\mu_{V}, \gamma_{V}\right)$, then $x_{r, s} \in U \Rightarrow \mu_{U}(x) \leq$ $r, s \geq \gamma_{U}(x)$ and $y_{p, q} \in V \Rightarrow p \leq \mu_{V}(y), q \geq \gamma_{V}(y)$. Then $1-q \leq 1_{\sim}-\gamma_{V}(y)$,

$$
\mu_{U}+\gamma_{U} \leq 1, \mu_{V}+\gamma_{V} \leq 1
$$

Now $U \cap V=0_{\sim} \Leftrightarrow \mu_{U} \wedge \mu_{V}=0, \gamma_{U} \vee \gamma_{V}=1$. From 3.1, $x_{r} \in \mu_{U}$ and $y_{1-q} \in 1-\gamma_{V}$. We will show that $\mu_{U} \wedge\left(1-\gamma_{V}\right)=0$ as follows. We have $\gamma_{U}(x)<1 \Rightarrow \gamma_{V}(x)=1 \Rightarrow 1-\gamma_{V}(x)=0$ which implies that $\left(\mu_{U} \wedge\right.$ $\left.\left(1 \sim-\gamma_{V}\right)\right)(x)=0 \sim$. Further if $\gamma_{V}(y)<1 \Rightarrow \gamma_{U}(y)=1 \Rightarrow 1-\gamma_{U}(y)=0$ since $\mu_{U}(y)+\gamma_{U}(y) \leq 1$, which implies that $\left(\mu_{U} \wedge\left(1 \sim-\gamma_{V}\right)\right)(y)=0 \sim$. Now take $z \in X$ such that $z \neq x$,y. If $\mu_{U}(z)=0$ there is nothing to prove. If $\mu_{U}(z) \neq 0$ then $\gamma_{U}(z)<1 \Rightarrow \gamma_{V}(z)=1 \Rightarrow\left(1 \sim-\gamma_{V}\right)(z)=0$ which implies that $\left(\mu_{U} \wedge\left(1 \sim-\gamma_{V}\right)\right)(z)=0 \sim$. Therefore $\mu_{U} \in \tau_{1}, 1_{\sim}-\gamma_{V} \in \tau_{2}$ such that $x_{r} \in \mu_{U}$, $y_{1-q} \in 1-\gamma_{V}$ and $\left(\mu_{U} \wedge\left(1 \sim-\gamma_{V}\right)\right)=0_{\sim}$. Hence, $\left(X, \tau, \tau_{2}\right)$ is $I B F$ pairwise IFG $\alpha T_{2}$.

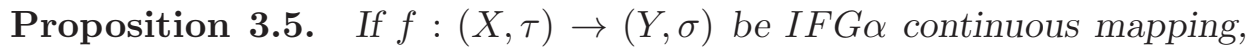

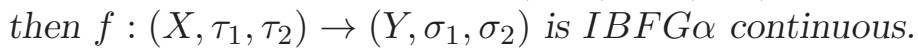

Proof. Let $U \in \sigma_{1}$ then there exists $V \in I^{Y}$ such that $(U, V) \in \sigma$. Since $f$ is IFGQ continuous, $f^{-1}(U, V) \in \tau$. This implies $\left(f^{-1}(U), f^{-1}(V)\right) \in \tau \Rightarrow$ 
$f^{-1}(U) \in \operatorname{tau}_{1} \Rightarrow f^{-1}(U)$ is $I F G \alpha$ open in $\tau_{1}$. Let $V_{1} \in \sigma_{2}$ then there exists $U_{1} \in I^{Y}$ such that $\left(U_{1}, V_{1}^{c}\right) \in \sigma$. Since $f$ is $I F G \alpha$ continuous, $\left.f^{-1}\left(U_{1}, V_{1}^{c}\right)\right) \in$

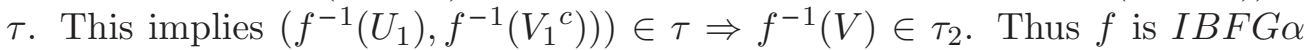
continuous.

\section{IF Pairwise $G \alpha$ Regular and Pairwise $G \alpha$ Normal Spaces}

Definition 4.1. (Pairwise Regular) An $I B F$ topological space $\left(X, \tau_{1}, \tau_{2}\right)$ is said to be $I F$ pairwise regular if for each $I F P x_{r, s} \in X$ and each $\tau_{i}$ closed set $F$ not containing $x_{r, s}$, there exist a $\tau_{i}$ open set $U$ and a $\tau_{j}$ open set $V$ such that $x_{r, s} \in U, F \subset V, U \cap V=0_{\sim}, i, j=1,2$ and $i \neq 2$.

Definition 4.2. (Pairwise $G \alpha$ Regular) An $I B F$ topological space $\left(X, \tau_{1}, \tau_{2}\right)$ is said to be $I F$ pairwise $G \alpha$ regular if for each $I F P x_{r, s} \in X$ and each $\tau_{i}$ closed set $F$ not containing $x_{r, s}$, there exist a $\tau_{i}$-GQ open set $U$ and a $\tau_{j}-G \alpha$ open set $V$ such that $x_{r, s} \in U, F \subset V, U \cap V=0_{\sim}, i, j=1,2$ and $i \neq 2$.

Proposition 4.1. An IBF topological space $\left(X, \tau_{1}, \tau_{2}\right)$ is said to be $I F$

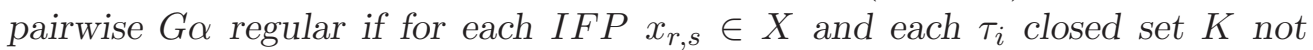

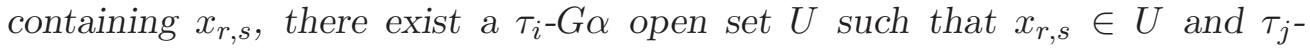
$\operatorname{G\alpha cl}(U) \cap K=0 \sim$.

Proof. Assume that $\left(X, \tau_{1}, \tau_{2}\right)$ is said to be $I F$ pairwise $G \alpha$ regular. Then from definition we have for each IFP $x_{r, s} \in X$ and each $\tau_{i}$ closed set $K$, there exist a $\tau_{i}-G \alpha$ open set $U$ and a $\tau_{j}-G \alpha$ open set $V$ such that $x_{r, s} \in U, K \subseteq V$ $U \cap V, i, j=1,2$ and $i \neq 2$. Since $\bar{K}$ is a $\tau_{i}$ open set containing $x_{r, s}$, then by

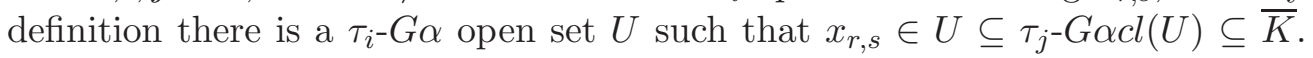
Hence $\tau_{j}-G \alpha c l(U) \cap K=0 \sim$.

Conversely, let $K$ be any $\tau_{i}$ closed set and $x_{r, s} \notin K$. Then there exists a $\tau_{i}-G \alpha$ open set $U$ such that $x_{r, s} \in U$ and $\tau_{j}$-Gacl $(U) \cap K=0_{\sim}$. Let $V=$ $\overline{\tau_{j}-G \alpha c l(U)}$. Then $V$ is a $\tau_{j}$-Ga open set $V$ such that $K \subseteq V, x_{r, s} \in U$ and $U \cap V=0_{\sim}$. Thus $\left(X, \tau_{1}, \tau_{2}\right)$ is said to be $I F$ pairwise $G \alpha$ regular.

Definition 4.3. A function $f:\left(X, \tau_{1}, \tau_{2}\right) \rightarrow\left(Y, \sigma_{1}, \sigma_{2}\right)$ is said to be $I F$ pairwise $G \alpha$ closed if the functions $f:\left(X, \tau_{1}\right) \rightarrow\left(Y, \sigma_{1}\right)$ and $f:\left(X, \tau_{2}\right) \rightarrow$

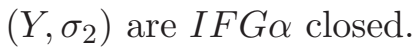

Definition 4.4. A function $f:\left(X, \tau_{1}, \tau_{2}\right) \rightarrow\left(Y, \sigma_{1}, \sigma_{2}\right)$ is said to be $I F$ pairwise $G \alpha$ continuous if the functions $f:\left(X, \tau_{1}\right) \rightarrow\left(Y, \sigma_{1}\right)$ and $f:\left(X, \tau_{2}\right) \rightarrow$ $\left(Y, \sigma_{2}\right)$ are $I F G \alpha$ continuous.

Proposition 4.2. Let $f$ be a pairwise continuous and $G \alpha$ closed function 
of a space $\left(X, \tau_{1}, \tau_{2}\right)$ on to a space $\left(Y, \sigma_{1}, \sigma_{2}\right)$. If $\left(X, \tau_{1}, \tau_{2}\right)$ is pairwise regular, $f^{(}\left(y_{p, q}\right)$ is $\tau_{1}$-compact and $\tau_{2}$, for each IFP $y_{p, q} \in Y$, then $\left(Y, \sigma_{1}, \sigma_{2}\right)$ is pairwise Ga regular.

Proof. Let $K$ be a $\sigma_{i}$-closed subset of $Y$ and let $y \in \bar{K}$. Then $f^{-1}(K)$ is a $\tau_{i}$ closed subset of $X$ and $f^{-1}(K) \cap f^{-1}\left(y_{p, q}\right)=0 \sim$. Since $X$ is $I F$ pairwise regular, for each $x_{r, s} \in f^{-1}\left(y_{p, q}\right)$ there exist $\tau_{j}$ open set $U_{k}$ and $\tau_{i}$ open set $V_{k}$ such that $f^{-1}(K) \subseteq U_{k}, x_{r, s} \in V_{k}$ and $U_{k} \cap V_{k}=0_{\sim}, i, j=1,2$ and $i \neq j$. The family $\left\{V_{k}: x_{r, s} \in f^{-1}\left(y_{p, q}\right)\right\}$ is a $\tau_{i^{-}}$open cover of $f^{-1}\left(y_{p, q}\right)$. Since $f^{-1}\left(y_{p, q}\right)$ is $\tau_{i}$-compact, there exist a finite sub cover of $\left\{V_{k}: x_{r, s} \in f^{-1}\left(y_{p, q}\right)\right\}$ say $V_{k}, k=$ $1,2,3, \ldots, n$ such that $f^{-1}\left(y_{p, q}\right) \subseteq \bigcup_{k=1}^{n} V_{k}$. Let $V=\bigcup_{k=1}^{n} V_{k}$ and $U=\bigcap_{k=1}^{n} V_{k}$. Then $V$ is $\tau_{i}$ open and $U$ is $\tau_{j}$ open. Also, $f^{-1}\left(y_{p, q}\right) \subseteq V, f^{-1}(K) \subseteq U$. Since $f$ is pairwise $G \alpha$ closed, there exist $V^{*} \in G \alpha O\left(Y, \sigma_{i}\right)$ and $U^{*} \in G \alpha O\left(Y, \sigma_{j}\right)$, $y_{p, q} \subseteq V^{*}, K \subseteq U^{*}, f^{-1}\left(V^{*}\right) \subset V^{*}$ and $f^{-1}\left(U^{*}\right) \subset U, i, j=1,2$ and $i \neq j$. Since $U$ and $V$ are disjoint sets $U \cap V=0_{\sim}$. Hence $\left(Y, \sigma_{1}, \sigma_{2}\right)$ is pairwise $G \alpha$ regular.

Definition 4.5. (Pairwise Normal) An $I B F$ topological space $\left(X, \tau_{1}, \tau_{2}\right)$ is said to be $I F$ pairwise normal if for each $\tau_{i}$ closed set $A$ and $\tau_{j}$ closed set $B$ such that $A \cap B=0_{\sim}$, there exist sets $U$ and $V$ such that $U$ is $\tau_{j}$ open set, $V$ is $\tau_{i}$ open set $V, A \subset U, B \subset V, U \cap V=0_{\sim}, i, j=1,2$ and $i \neq 2$.

Definition 4.6. (Pairwise $G \alpha$ Normal) A space $\left(X, \tau_{1}, \tau_{2}\right)$ is said to be pairwise $G \alpha$ normal if for any $\tau_{i}$ closed set $A$ and $\tau_{j}$ closed set $B$ disjoint from

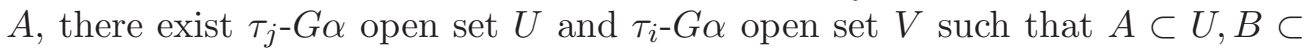
$V, U \cap V=0 \sim, i, j=1,2$ and $i \neq 2$.

Proposition 4.3. Let $f:\left(X, \tau_{1}, \tau_{2}\right) \rightarrow\left(Y, \sigma_{1}, \sigma_{2}\right)$ be a pairwise continuous and pairwise $G \alpha$ closed surjection. If $\left(X, \tau_{1}, \tau_{2}\right)$ is pairwise normal then $\left(Y, \sigma_{1}, \sigma_{2}\right)$ is pairwise $G \alpha$ normal.

Proof. Assume that $\left(X, \tau_{1}, \tau_{2}\right)$ is pairwise normal. Let $A$ and $B$ be two disjoint subsets of $Y$ such that $A$ is $\sigma_{i}$-closed and $B$ is $\sigma_{j}$-closed. Since $f$ is pairwise continuous, $f^{-1}(A)$ is $\tau_{i}$-closed and $f^{-1}(B)$ is $\tau_{j}$-closed sets such that $f^{-1}(A) \cap f^{-1}(B)=0 \sim$. Since $X$ is pairwise normal, there exist disjoint sets $U$ and $V$ such that $f^{-1}(A) \subseteq U$ and $f^{-1}(B) \subseteq V, U$ is $\tau_{i}$-open and $V$ is $\tau_{j}$-open. Now $f$ is pairwise $G \alpha$ closed, there exist $\sigma_{j^{-}} G \alpha$ open set $L$ and $\sigma_{i^{-}} G \alpha$ open set $M$ such that $A \subset L, B \subset M$ with $f^{-1}(L) \subseteq U$ and $f^{-1}(M) \subseteq V$. Since $U \cap V=0_{\sim}$, we have $L \cap M=0_{\sim}$. Hence $\left(Y, \sigma_{1}, \sigma_{2}\right)$ is pairwise $G \alpha$ normal. 


\section{Conclusion}

Thus, we have discussed the nature of some pairwise $G \alpha T_{K}$ separation axioms via bifuzzy $G \alpha$ open sets in IFTS. Pairwise $G \alpha$ regular, pairwise $G \alpha$ normal spaces are studied and their inter relations are proved. This may be very useful when we study about how open sets are separated by closed sets in intuitionistic bifuzzy topological spaces. This study may bring an intuition among the researchers to develop pairwise separation axioms in other generalized closed sets in intuitionistic bifuzzy Topological Spaces.

\section{Acknowledgments}

The author pays her sincere thanks to the unknown referees for their guidance to improve their work.

\section{References}

[1] K.T. Atanassov, Intuitionistic Fuzzy Sets, Fuzzy Sets and Systems, 20 (1986), 87-96.

[2] A.S. Abu Safiya, A.A. Fora, M.W. Warner, Higher separation axioms in bifuzzy topological spaces, Fuzzy Sets and Systems, 79 (1996), 367-372.

[3] A.S. Abu Safiya, A.A. Fora, M.W. Warner, Fuzzy separation axioms and fuzzy continuity in fuzzy bitopological spaces, Fuzzy Sets and Systems, 62 (1994), no. 3, 367-373.

[4] Amit Kumar Singh, Rekha Srivastava, Separation axioms in intuitionistic fuzzy topological spaces, Hindawi Publishing Corporation, Advances in Fuzzy Systems, Volume (2012), Article ID604396, 7 pages.

[5] S. Bayhan, D. Coker, On fuzzy separation axioms in intuitionistic fuzzy topo logical spaces, BUSEFAL, 67 (1996) 77-87.

[6] S. Bayhan, D. Coker, On $T_{1}$ and $T_{2}$ separation axioms in intuitionistic fuzzy topological spaces, J. Fuzzy Mathematics, 11, No. 3 (2003), 581-592.

[7] D. Coker, An introduction to intuitionistic fuzzy topological spaces, Fuzzy Sets and Systems, 88 (1997), 81-89. 
[8] K. Chandrasekhara Rao, V. Subha, A note on the associated bitopological space $\left(X, \tau_{1}^{*}, \tau_{2}^{*}\right)$, Int. J. of Math. Analysis, 7, No. 7 (2013), 323-330.

[9] A.A. Fora, Separation axioms for fuzzy spaces, Fuzzy Sets and Systems, 33 (1989), 59-75.

[10] F. Gallego Lupianez, Hausdorffness in intuitionistic fuzzy topological spaces, Mathware and Soft Computing, 10 (2003), 17-22.

[11] C.S. Gowri, D. Kalamani, R. Dhavaseelan, Separation axioms via intuitionistic fuzzy generalized open sets, Accepted.

[12] D. Kalamani, K. Sakthivel, C.S. Gowri, Generalized alpha closed sets in intuitionistic fuzzy topological spaces, App. Math. Sci., 6, No. 94 (2012), 4691-4700.

[13] J.C. Kelly, Bitopological Spaces, Proc. London Math. Soc., 13 (1963), 71-89.

[14] T.M. Nour, A note on five separation axioms in bitopological spaces, Indian J.pure appl. Math., 26, No. 7 (1995), 669-674.

[15] L.A. Zadeh, Fuzzy Sets, Infor. and Control, 9 (1965), 338-353. 
